Research Article

\title{
Design of Enterprise Management System Based on Edge Computing Architecture
}

\author{
Yongzhong Yang, ${ }^{1}$ Zheng Mei, ${ }^{1}$ Benxia Zheng, ${ }^{2}$ and Shuoli Qiu ${ }^{1}{ }^{1}$ \\ ${ }^{1}$ School of Sichuan University, Chengdu Sichuan 610041, China \\ ${ }^{2}$ Sichuan Vocational College of Finance and Economics, Sichuan Chengdu 610101, China \\ Correspondence should be addressed to Shuoli Qiu; hujiheng@stu.scu.edu.cn
}

Received 9 February 2021; Revised 23 March 2021; Accepted 24 April 2021; Published 3 May 2021

Academic Editor: Hsu-Yang Kung

Copyright (c) 2021 Yongzhong Yang et al. This is an open access article distributed under the Creative Commons Attribution License, which permits unrestricted use, distribution, and reproduction in any medium, provided the original work is properly cited.

\begin{abstract}
In the current enterprise management system, the coupling relationship between enterprise management and operation and maintenance database is not considered, which leads to many system vulnerabilities, slow running speed, and long response time. An enterprise management system based on edge computing architecture is designed. According to the characteristics of mobile edge computing, we design the architecture of the edge computing platform, integrate the MEC virtualization infrastructure layer, MEC platform layer, and MEC application layer, construct the edge computing model, reflect the coupling relationship between enterprise management and operation and maintenance database, calculate the delay vector of the enterprise management server, and complete the parameter calculation of enterprise management and operation and maintenance system. This paper analyzes the feasibility, objectives, and users and functional requirements of the enterprise management system, designs the functional structure of the system, realizes the design of the public module, function menu module, and login module, adopts the SQL database management system, and designs the database and data table, so as to realize the design of the enterprise management system based on edge computing architecture. Experimental results show that the proposed method has fewer system vulnerabilities and can effectively speed up the system operation speed and shorten the system response time.
\end{abstract}

\section{Introduction}

Enterprise informatization is an important means to improve the speed of modernization. In order to speed up the informatization construction and modernization process of the government and enterprises, government departments need to introduce the office automation system to improve the office efficiency and transparency of government staff [1]. It is not enough for an enterprise to only introduce a system. It also needs to introduce a complete and comprehensive information management platform, increase the depth and strength of enterprise informatization, and strive to make the enterprise develop healthily and rapidly in the fierce international market competition under the guidance of information, so as to play a positive role in the industrialization and modernization of the enterprise [2]. The introduction of the information management platform is the best choice for enterprises and companies, especially for production and sales enterprises, to realize their information strategy. Through the introduction of the information enterprise management platform, we can introduce the most advanced modern management ideas and methods in the world. Therefore, the enterprise information management platform is a powerful tool to help enterprises develop, make enterprises step into the modern enterprise management mechanism, and strengthen the market competitiveness of enterprises. Although there is a great demand for information management software in the current market, the information management software obtained according to the previous development method has to be redeveloped when the business changes or the demand changes slightly, resulting in a huge waste of human and financial time [3]. Therefore, how to improve the software productivity and software quality of the information management platform, on the basis of realizing complex software requirements and improving software quality, to improve software 
productivity has become the consistent pursuit of many information management platform developers.

The process of enterprise management informatization is accelerating, and the application of the information system has a profound impact on enterprise management. The competition among enterprises is becoming more and more fierce. More and more enterprises realize the importance of information and management system in improving the enterprise management level, reducing enterprise operating costs, improving service quality, and meeting customer needs. At present, the research on the enterprise management system has also made great progress. Ducq et al. [4] proposed the design of the enterprise performance measurement system, using a group of specific performance indicators, and grouping them in the coherent system of the performance measurement system. The design objectives and basis are different, and each method has its advantages and disadvantages. It can measure the performance optimally. In this paper, the main concepts of various methods are described. Introduce as many new methods as possible, describe them in detail, and compare them so that decision makers can choose one or more methods that suit their needs. The system can effectively achieve the global goal of PMS design and implementation. The authors of [5] proposed the management design of the last planner system in the enterprise construction stage, studied and discussed the designers, engineers and managers sharing their knowledge and solving design related problems during the site meeting, the influence of using LPS in the design stage on the site meeting discussion in the construction stage, and the video records of 17 site meetings in the BIM transformation project to share their professional knowledge to solve the outstanding problems. This method can develop more collaborative design management methods and practices. However, the above method does not consider the coupling relationship between enterprise management and operation and maintenance database, which leads to many system vulnerabilities, slow running speed, and long response time. The growing computing power on intelligently connected things and devices requires the decentralization of cloud computing to avoid unnecessary latency and take full advantage of the available computing power at the edge of the network. Literature [6] analyzes existing works to identify their role in decentralized cloud and future computing development. Growing service demands for terminal devices may overwhelm installed MECs, and cost constraints limit the increase in installed MEC computing and data storage capacity. Literature [7] divides the device-enhanced MEC mechanism into computational offload mechanism and cache mechanism and further subdivides the offload mechanism and cache mechanism according to the target performance target. In addition, literature [7] identifies the main limitations of the existing device-enhanced MEC mechanism and outlines future research directions.

The Mobile Cloud Computing (MCC) [8] is a precedent for edge and fog computing. It defines the concept of a proximal cloud, which provides tighter computing power to avoid latency on the mobile devices it serves. Many writers have taken advantage of this connection. Examples are Bilal et al. [9], Satyanarayanan [10], and Satyanarayanan et al. [11]. The first steps towards decentralization of cloud computing are being achieved through the emergence of fog [12] and edge computing [13]. These are believed to be rooted MCC $[9,11]$. The OpenFog Consortium in its reference architecture refers to the fact that fog computing is often misnamed as edge computing and debates the different levels of cloud interaction, layers, and aspects handled. A recent publication of the OpenFog Consortium blog expands this definition, arguing that fog computing is a continuum or range from cloud computing and edge computing to device computing.

To solve the above problems, the enterprise management system based on edge computing architecture is designed. By designing the edge computing platform architecture and integrating the MEC virtualization infrastructure layer, MEC platform layer, and MEC application layer, the edge computing model is constructed, and the server delay vector is calculated. This paper analyzes the requirements of the enterprise management system, designs the system function structure, realizes the design of the public module, function menu module, and login module, adopts the SQL database management system, designs the database and data table, and realizes the design of the enterprise management system based on edge computing architecture. The system has fewer loopholes, which can effectively speed up the operation of the system and shorten the response time of the system.

\section{Edge Computing Technology}

2.1. Concept and Characteristics of Edge Computing. Mobile edge computing (MEC) technology refers to the deployment of general servers on the wireless access side, which provides it with cloud computing capabilities for the wireless access network [14]. MEC technology allows direct mobile communication between edge core network and mobile terminal enterprise users. Each mobile terminal enterprise user has its corresponding MEC core network. The wireless access network thus has the ability of low delay, high bandwidth transmission, and open wireless network information perception, avoiding bottlenecks and system failures. At the same time, the computing task and data sink, i.e., localization deployment, can effectively reduce the computing load and storage load of the mobile system, so as to realize the purpose of optimizing the operation cost of the mobile network. In addition, mobile operators can form a new industrial chain with mobile cloud platform, application developers, and network equipment manufacturers to cooperate and benefit together through the mobile edge computing platform.

According to the white paper published by the European Telecommunication Standardization Association, the characteristics of mobile edge computing are as follows:

(1) Locality: MEC platform can access local resources and run independently from other parts of the core network. This isolation from other parts of the network also makes the MEC network less vulnerable to external attacks. 
(2) Proximity: mobile edge computing intelligent base station is deployed in the nearest location from the mobile terminal, which has the advantage of acquiring, analyzing, and mining big data. Accordingly, it is also beneficial to the need for computer computing power.

(3) Reduce latency: the mobile edge computing intelligent base station service is deployed in the nearest location from the mobile terminal equipment, which can isolate the core network from the mobile network data. Therefore, this isolation brings high bandwidth, low latency, and high-quality enterprise user experience.

(4) Location awareness: distributed devices under the jurisdiction of the mobile edge computing intelligent base station can use low-level signaling to realize information sharing with the intelligent base station, so as to find the location of each distributed device.

(5) Network context information: mobile edge computing can be applied to the business model of some specific applications, which can benefit from both application service providers and application service enterprise users. Based on the real-time information of the wireless access network, the congestion degree and bandwidth occupation degree of the wireless cellular network can be estimated, which will assist such applications to make decisions to improve the quality of service.

\subsection{Architecture Design of the Edge Computing Platform.} The MEC system platform in this paper integrates the MEC virtualization infrastructure layer, MEC platform layer, and MEC application layer. The architecture of the mobile edge computing platform is shown in Figure 1.

In the MEC virtualization infrastructure layer, edge devices are connected through a variety of networks (such as mobile cellular network, local network, and external network), and enterprise management data interaction is realized through a variety of communication methods. In the MEC platform layer, virtualization infrastructure and MEC applications are integrated. Virtualization infrastructure has the function of infrastructure service, which provides diversified and efficient runtime and hosting environment for the application of the enterprise management data program [15]. Its infrastructure service controller can control the work of the enterprise management platform, give full play to its application advantages, and provide more secure and reliable enterprise management application resources and safe program running environment. In this facility, a virtual device application is installed in the virtual device, which can run on top of the enterprise management infrastructure service. In the above system, the enterprise management platform service facilities have strong compatibility in the application platform services and are suitable for a variety of services to smoothly complete the collection, analysis, application, and processing of enterprise management data.
The MEC application layer is an important layer in the MEC platform, including the ME host set in operator network architecture and ME algorithm model necessary for enterprise management ME application, which can allocate MEC tasks and control the operation of the whole enterprise management system [16]. By using MEC technology, it can provide highly distributed computing environment and information storage function in the enterprise information management platform, so as to effectively reduce network delay and improve the quality of enterprise user experience.

2.3. Edge Computing Model. In edge computing, the algorithm can directly reflect the coupling relationship between different parameters in the enterprise management database and operation and maintenance database and edge computing system. In practical application, analyzing the requirements of enterprise management application scenarios and mapping them to the parameters of the classification model can help enterprise users quickly select the best edge computing system of target application scenarios. The structural characteristics of the edge computing model are shown in Figure 2.

When calculating the enterprise management and operation and maintenance system, it includes the following steps: firstly, obtain the data information in the enterprise management database and the enterprise operation and maintenance system database information, the data information includes the enterprise management ability and operation level, and the enterprise management ability data includes the load data and transfer data. Operation level data includes comprehensive index data, equipment level data, and equipment operation status data [17]. When measuring enterprise management and operation, we usually measure the resource consumption required by the data enterprise application server to start a session, and delay information about the start of the MEC server.

Next, by constructing the resource consumption and enterprise application service delay model required by the enterprise application server to start the session, the session delay vector $d_{i j m}$ of the enterprise application service can be further calculated. When calculating the session delay vector, the model is constructed as follows:

$$
d_{i j m}\left(c_{i}\right)=\frac{\varphi_{i}}{1+\sigma_{i} e^{-\omega_{i} c_{i}}} .
$$

In formula (1), $d_{i j m}$ is the time delay for data calculation when the program $i$ is running in the $j m$ enterprise management server in the mobile edge computing network $m . \varphi_{i}$, $\sigma_{i}$, and $e^{-\omega_{i} c_{i}}$ are represented as the first, second, and third regression parameters, respectively, $i$ is the program startup session, and $c_{i}$ is the amount of CPU resources required by the edge device for program startup calculation $i$.

When calculating the delay vector of the enterprise management server, for the convenience of calculation, it is represented by the letter $d_{i, m j m, p}$, and the formula calculation model is 


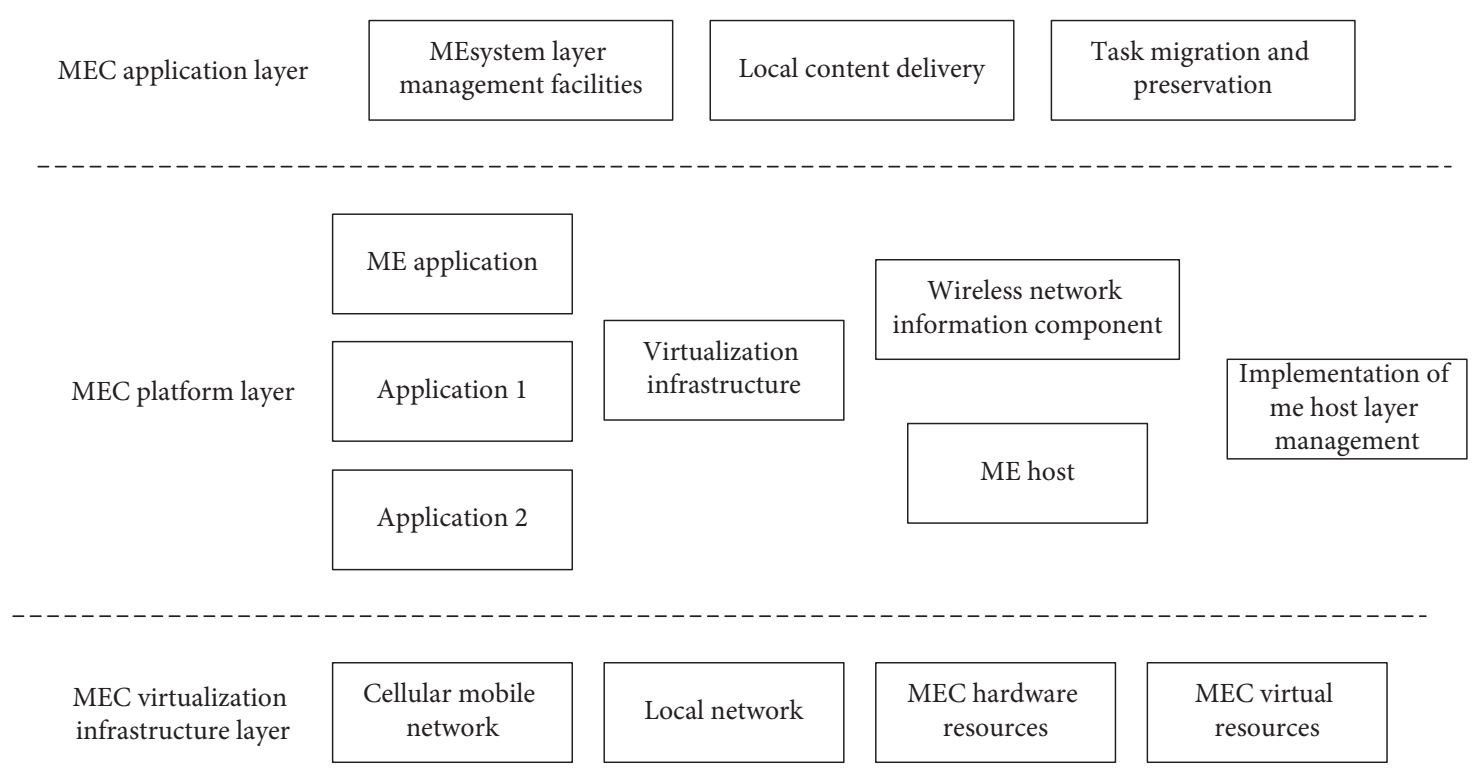

FIgUre 1: Architecture of the edge computing platform.

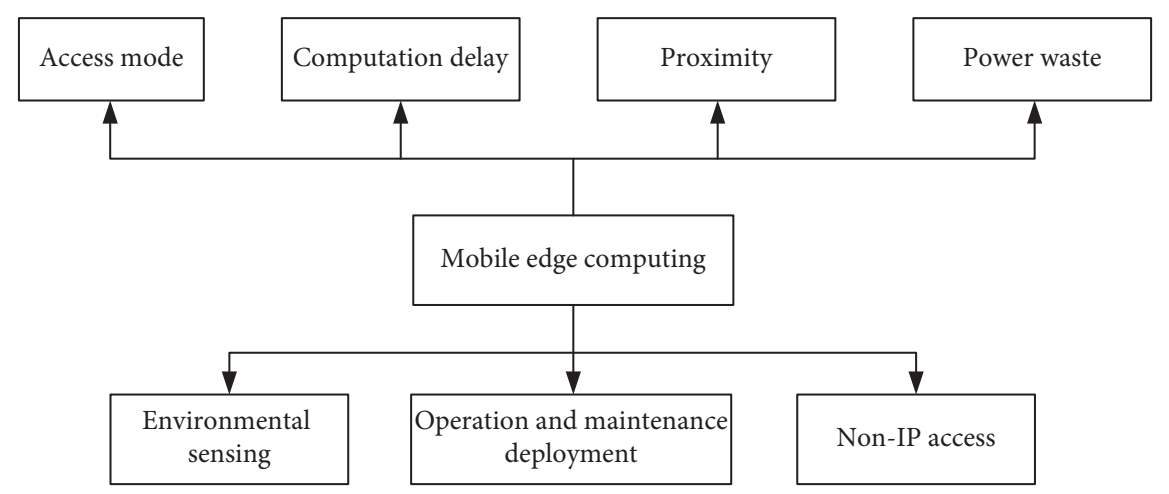

Figure 2: Structural features of the edge computing model.

$$
d_{i, m j m, p}=\frac{1}{2}\left[\left(\mathrm{RTT}_{i, j m, p}-\mathrm{RTT}_{i, m, p}\right)-\left(T_{j m, s}-T_{j m, a}\right)\right] .
$$

In formula (2), $d_{i, m j m, p}$ is the network delay of enterprise management operation. More specifically, the delay is the time delay for connecting the core switch $m$ and the enterprise management server $j m$ to start the session $i$ in the enterprise management, in the $m$ information communication network, and in the $p$ data communication path [18]. $\mathrm{RTT}_{i, m, p}$ is the round-trip delay for the $i$ session between the controller used in the network configuration and the $m$ switch in the $p$ path. $\mathrm{RTT}_{i, j m, p}$ is the round-trip delay of the $i$ session between the controller used by the network configuration and the enterprise management server $j m$ in the $p$ path. $T_{j m, s}$ is the sending time of the enterprise management server $j m$ message, and $T_{j m, a}$ is the arrival time of the message.
When calculating the delay vector of the enterprise management server group, it is represented by $d_{i, m n, p}$, and the model formula is

$$
d_{i, m n, p}=T_{\alpha}-T_{s}-\frac{1}{2}\left(\mathrm{RTT}_{i, n, p}-\mathrm{RTT}_{i, m, p}\right) .
$$

In formula (3), $d_{i, m n, p}$ is the network delay, that is, in the $p$ communication path, and the core switch $m$ is connected to the core switch $n$ to start the network delay of the session $i$. $R T T_{i, m, p}$ is the round-trip delay of the $i$ session between the controller and switch $m$ in the $p$ communication path, and $R T T_{i, n, p}$ is the round-trip time of the $i$ session between the controller and switch $n$ in the $p$ path extension. $T_{\alpha}$ is the time from the controller to switch $m$, and $T_{s}$ is the time sent by switch $n$.

Then, express the relationship between the session delay vector, the enterprise management server delay vector, and the enterprise management server group delay vector through the objective function. The function is expressed as 


$$
O_{\text {total }}=\sum_{i=1}^{N_{g m}} \sum_{j=1}^{N_{s m}} \sum_{m=1}^{N_{m}}\left[\alpha_{i}\left(\sum_{p=1}^{N_{p m}} d_{i, m j m, p} x_{i, m j m, p}\right)\right]+\left[\beta_{i}\left(\sum_{p=1}^{N_{p, m n}} d_{i, m n, p} y_{i, m n, p}\right)+\gamma_{i} d_{i j m} z_{i j m}\right] .
$$

In formula (4), $N_{m}$ is the number of networks $m$ used for mobile edge computing, $N_{s m}$ is the number of enterprise management servers $s$ used in the mobile edge computing network $m$, and $N_{g m}$ is the mobile edge computing network $m$, where the enterprise management server starts the number of sessions $i, N_{p m}$ is the number of $p$ paths between the switch and the enterprise management server in the mobile edge computing network $m$, and $N_{p, m n}$ is the number of $p$ paths between the mobile edge computing network $m$ and $n . \alpha_{i}, \beta_{i}$, and $\gamma_{i}$ are expressed as the first, second, and third weighting factors of the objective function, where $\gamma_{i}$ is obtained according to $1-\alpha_{i}-\beta_{i}$, and $x_{i, m j m, p}, y_{i, m n, p}$, and $z_{i j m}$ are expressed as the first, second, and third decision variables. Through the above formula calculation, the calculation of various parameters in the enterprise management and operation and maintenance system is realized.

\section{Requirement Analysis of the Enterprise Management System}

Requirement analysis refers to the detailed investigation of the objects to be dealt with, clear user needs, to determine the purpose of new system functions [19]. Requirement analysis is the first stage of the database structure design, and it is also a very important stage. In this stage, it is mainly to investigate, collect, and analyze the information requirements, processing requirements, and security and integrity requirements of users in data management. Information requirement refers to the content and nature of information users need to obtain from the database.

3.1. System Feasibility Analysis. In order to realize the better management of the enterprise, the enterprise needs to have a good management system, realize the scientific and effective management of employees, check the inventory status in real time, adjust the quantity of inventory materials, supply materials for the production of products, and feed back the completion of the plan in time after the completion of the products. The enterprise management system is designed based on the edge computing framework. This system integrates the database technology and edge computing technology, makes the database system an important organic part, and realizes the organic combination of database technology and edge computing technology. The system can be analyzed from three aspects: economic feasibility, technical feasibility, and operation feasibility:

(1) Operation feasibility: because this system is an information system developed for enterprise employees, although these employees may not have used a similar system, with the friendly interface of Windows and the good security settings of this system, users can quickly master the operation method of the system under the guidance and help of the navigation column of the enterprise management website. At the same time, users can learn to use the system correctly according to the detailed "user manual."

(2) Technical feasibility: from the current popular database development and management software point of view, for the information management system related to the database, using edge computing architecture development and MySQL in the database, is the most appropriate solution in practical application. The development members are very skilled in using this technology and can develop this system well from the technical point of view.

(3) Economic feasibility: the main way to improve the management level is to update the ideas of managers and enhance the scientific understanding of management activities [20]. At the same time, the use of mature information technology, the development of the network office system, is an effective measure to deepen the reform of enterprise management. Enterprises need to build their own company management personnel management system. In order to make the system development smoothly, it provides a lot of financial support.

3.2. System Objective Requirement Analysis. As an enterprise personnel management platform, the enterprise management system should meet the following goals in design:

(1) Based on mature technology to create the system: whether the construction goal of the enterprise management system can be achieved and whether the whole investment of the system can play its due benefits will ultimately depend on whether the system is reliable and practical. Therefore, mature and reliable technology should be adopted in the system, and the design principle and implementation method of productization should be implemented.

(2) Conducive to the improvement of the information resource-sharing rate: the enterprise management system is based on LAN or Internet, striving to achieve high-level information resource sharing and cross-platform information resource access. Due to the different platforms and databases of network information systems and resource subsystems, the system structure must have the mechanism of crossplatform access to different data sources to solve the information sharing of different resource subnets and improve the occupancy rate of information resource sharing.

(3) The system is simple, easy to use, and maintain: the design of the enterprise personnel management 
system should meet the needs of enterprise management, with complete and practical functions and an easy to learn, friendly, and clear interface. The network structure should be simple, clear, and easy to manage. These measures can fully realize the management functions of the product company. It provides a convenient and quick information query function for users.

(4) Safe and reliable performance: the enterprise management system should have a safe and efficient communication mechanism, identity authentication, and authority check to solve the security and confidentiality problems of the system and prevent information leakage and illegal intrusion into confidential information.

(5) High quality information service: on the basis of information standardization and normalization, we should make a reasonable layout of information and provide high-quality and efficient business management and transaction processing. At the same time, the system runs stably, safely, and reliably.

3.3. System User Analysis. The reasonable design of user rights is the basis to ensure the normal operation of the system and the maximum efficiency of the system. The personnel management system involves trade secrets to a certain extent, so it has higher requirements for the user authority design [21]. The system administrator is the owner of the highest authority, who not only can complete the operation of the general administrator but also can manage the information of the general administrator. Ordinary users can only log in to the system for basic operation after the administrator registers for them. System users include ordinary users, general administrators, and general administrators.

Functions of ordinary users: the functions of ordinary users are to manage the basic information of their own employees, modify their own passwords, query basic information of the department, query the type of attendance information, query their own daily attendance and monthly attendance information, query their own basic salary and subsidies information, query your own past and current salary information, manage your own personal communications and query company communications, query rewards and punishments information, query job change information, and send and receive emails.

Functions of general administrators: general administrators can query the basic files of management employees, but cannot modify the passwords of other ordinary employees, manage basic information of the department, manage attendance information, query the daily attendance and monthly attendance of themselves and other ordinary employees information, manage basic salary and subsidy information, generate employee salary, manage employee position change information, manage files, manage employee reward and punishment information, manage personal address book, and recruit and train employee management.
The function of the system administrator: the function of the system administrator is to query the basic information of the management staff, but cannot modify the passwords of other ordinary employees and the basic information of the management department, manage the attendance information, query the daily attendance and monthly attendance of themselves and other ordinary employees information, manage basic salary and subsidy information, and generate employee salaries, administrator information management, system event query, system decision-making and statistics, security management, database management, and file management.

3.4. System Function Analysis. The function of the system must be able to meet the user's information requirements, processing requirements, and security and integrity requirements. The goal of the enterprise management system is to build a brand-new modern enterprise management system according to the requirements of enterprise management, realize the automation, digitization, and intellectualization of employee management, purchase, warehouse management, distribution management, and other activities through computer, network, and other modern science and technology, and build an information platform for enterprise management [22]. This paper proposes that the enterprise management system based on edge computing technology mainly includes three modules, namely, enterprise personnel management, enterprise inventory management, and system maintenance. The enterprise personnel management system also includes employee management, department management, attendance management, salary management, and other submodules. The enterprise inventory management also includes commodity warehousing management, commodity inventory management, commodity sales management, data statistics and reports, and other submodules.

(1) The main work content of employee management is to add, modify, delete, and query employee information, as well as detailed records of employee transfer, including the adjustment of department, position and title, and employee turnover.

(2) The main work of department management is to add, modify, delete, and query departments.

(3) The main work content of attendance management is to add and modify daily attendance records. It focuses on the function of adding and modifying daily attendance records according to all employees, department employees and selected employees, so as to facilitate user operation. In addition, it can add, modify, delete, and query employees' daily leave information.

(4) The main work content of salary management is batch addition, modification, deletion, and query of monthly salary information. In addition, it focuses on the export function of salary data, which can be exported in the form of Excel file or online banking file. 
(5) Commodity warehousing management is the planning and organization of various business activities such as unloading, checking, acceptance, and handling warehousing procedures when receiving warehousing goods according to the commodity warehousing voucher. Its basic requirement is to ensure that the quantity of goods in storage is accurate and the quality meets the requirements. Its main work content is goods storage and storage query.

(6) Inventory management includes warehouse management and inventory control. Warehouse management includes warehouse planning, goods in and out management, and warehouse quality management. Inventory control mainly refers to the control of inventory quality. Its main work content is commodity lending, commodity lending query, commodity return, commodity return query, commodity inventory, and inventory query.

(7) Commodity sales management is a software used to help users manage the commodity sales process. Users can easily realize the management of commodity sales process by inputting the goods, orders, sellers, and other data involved in the sales process. Its main work content is commodity sale and sale inquiry.

(8) Statistical report is to provide basic statistical data from the bottom to the top according to unified form, unified submission procedure, and report time. Its main work content is in and out of warehouse day statistics, in and out of warehouse year statistics, out of warehouse report, and inventory report.

The system maintenance module is a functional module mainly for maintaining system security. Its main work contents are operator management, password modification, operator permission setting, data backup, data recovery, data cleaning, log viewing, and log cleaning.

\section{Enterprise Management System Design}

The goal of the enterprise management system design is to design a practical, easy-to-use, extensible, easy to maintain, stable, and efficient enterprise management system to adapt to the current situation of enterprise management and to design a general enterprise management system suitable for the current management situation of most enterprises through this system [23]. The design process of the enterprise management system must strictly abide by the software engineering development process, and the design should follow the principles of practicability, security, ease of use, efficiency, stability, easy maintenance, and scalability of the system.

4.1. System Function Structure Design. According to the analysis of the functional requirements of the enterprise management system, the enterprise management system is divided into three functional modules, namely, enterprise personnel management, enterprise inventory management, and system maintenance. The enterprise personnel management system also includes employee management, department management, attendance management, salary management, and other submodules. The enterprise inventory management also includes commodity warehousing management, commodity inventory management, enterprise management, commodity sales management, data statistics and reports, and other submodules. The functional structure of the system is shown in Figure 3.

\subsubsection{Enterprise Personnel Management Module. Enterprise} personnel management is one of the information management systems that must be owned by modern enterprises and institutions to manage employees, wages, and daily attendance. It should include the addition, deletion, modification and check of personnel information, personnel transfer and resignation, printing and output of personnel information, daily attendance management, wage management, and welfare management. The enterprise personnel management module includes employee management, department management, attendance management, salary management, and other four submodules. The main work of employee management is to add, modify, delete, and query employee information, as well as detailed records of employee transfer, including the adjustment of department, position and professional title, and employee turnover. The main work content of department management is the addition, modification, deletion, and query of department staff. It mainly includes two submodules: adding department information and modifying department information. The main work content of attendance management is to add and modify daily attendance records. It focuses on the function of adding and modifying daily attendance records according to all employees, department employees and selected employees, so as to facilitate users to add, modify, delete, and query employees' daily leave and leave information. It mainly includes three submodules: attendance information setting, adding and modifying attendance records, and employee leave. The main work content of salary management is batch addition, modification, deletion, and query of monthly salary information. In addition, it focuses on the export function of salary data, which can be exported in the form of Excel file or online banking file. It mainly includes three submodules: salary information management, overdue data processing, and salary export.

\subsubsection{Enterprise Purchase, Sale, and Inventory Management} Module. The enterprise purchase, sale, and inventory management module can realize the information management of the enterprise's purchase, sale, inventory, and other businesses. It is a necessary condition for the steady development of small- and medium-sized enterprises in the modern society. It can improve the management level and work efficiency of enterprises and minimize the mistakes of manual operation. The enterprise purchase, sale, and inventory management module includes four submodules: 


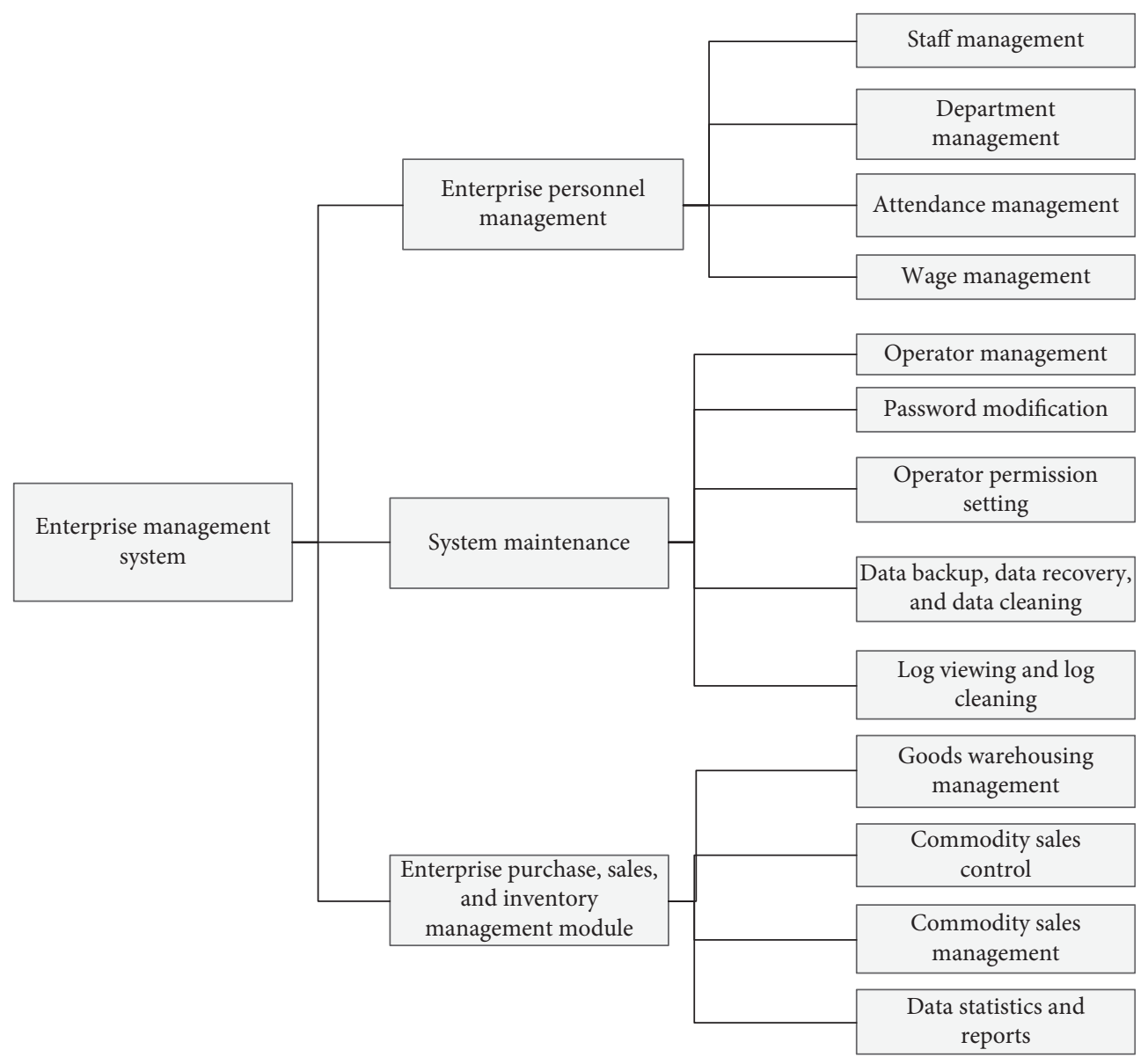

FIGURE 3: System function structure diagram.

commodity storage management, commodity inventory management, commodity sales management, and data statistics and reports. Before using this system, enterprises need to establish the basic information of enterprises in the system, including commodity information setting, warehouse information setting, and initial inventory setting. Commodity warehousing management is the plan and organization of various business activities such as unloading, checking, acceptance, and handling warehousing procedures when receiving warehousing goods according to the commodity warehousing voucher. Its basic requirement is to ensure that the quantity of goods in storage is accurate and the quality meets the requirements. It mainly includes goods warehousing, warehousing query, and so on. Inventory management includes warehouse management and inventory control. Warehouse management includes warehouse planning, goods in and out management, and warehouse quality management. Inventory control mainly refers to the control of inventory quality. The commodity inventory management module is mainly to complete the work of commodity lending, commodity lending query, commodity return, commodity return query, commodity inventory, inventory query, etc. Commodity sales management is a module used to help users manage the commodity sales process. Users can easily realize the management of commodity sales process by inputting the goods, orders, sellers, and other data involved in the sales process. The commodity sales management is mainly to complete the commodity sales and sales inquiry. Statistical report is to provide basic statistical data from the bottom to the top according to unified form, unified submission procedure, and report time. The data statistics and report module is mainly used to complete the daily statistics of in and out of warehouse, annual statistics of in and out of warehouse, out of warehouse report, inventory report, etc.

4.1.3. System Maintenance Module. The system maintenance module is a functional module mainly to maintain system security, which mainly includes operator management, password modification, operator permission setting, data backup, data recovery, data cleaning, log viewing, and log cleaning.

\subsection{Database Design}

4.2.1. Data Table Design. According to the system function structure design, a number of data tables are established. They are employee information table, department table, salary table, attendance record table, inventory information table, commodity warehousing information table, commodity outbound table, and other related data tables. 
(1) Employee information table: employee information table records the basic information of employees, including employee ID number, employee name, employee gender, employee ID card number, employee birth date, employee participation time, employee entry time, staff working age, and other information

(2) Department table: it is mainly used to record the department information of an enterprise, including department number, department name, department manager, department address, and department telephone number

(3) Payroll: the payroll is used to store the detailed salary information of each employee every month, including the salary month, employee number, employee name, employee basic salary, employee overtime pay, employee seniority salary, employee pension insurance, employee medical insurance, employee salary payable, employee salary deduction, and employee actual salary

(4) Attendance record table: it is used to record the daily attendance of employees, including employee number, employee name, employee commuting situation, employee commuting time, date, whether abnormal, abnormal time, and other information

(5) Inventory information table: the inventory information table is used to store the relevant information of commodity inventory, including commodity number, commodity name, commodity specification, commodity unit, commodity receipt quantity, commodity unit price, and commodity receipt date

(6) Commodity warehousing information table: it is used to store relevant information of commodity warehousing, including commodity number, commodity name, supplier number of commodity, supplier name of commodity, commodity specification, commodity quantity, commodity unit price, commodity amount, commodity warehousing date, commodity warehouse year, commodity warehousing month, handler, modifier, and modification date

(7) Commodity delivery table: commodity delivery table is used to store the delivery information of commodities, including delivery number, commodity number, commodity name, unit of measurement, quantity of delivered commodities, delivery date, delivery year, delivery month, delivery company, delivery person, handler, modifier, and modification date

4.2.2. Common Module Design. When designing the system, those functions that may be reused should be written as general procedures or functions and stored in standard modules. This not only reduces the amount of code but also facilitates future maintenance. In addition, in the standard module, you can also define some common variables, which can facilitate the mutual transfer of values between the functional windows. Before using a standard module, you should first create it by selecting the "Project" "Add Module" command to create a new module, using the default name Module 1.

4.2.3. Function Menu Design. The realization of various functions of the system is realized through the operation of the menu and supports the operation of the keyboard and the mouse at the same time. The menu has a first-level menu, a second-level menu, and a third-level menu. The menu of this system is mainly designed through the menu editor. First, select the "Project"/"Menu Editor" command in the project, open the "Menu Editor" dialog box, and design in it. After the menu is designed, you need to add code to its Click event, that is, the code to call each functional form. Use the Load statement to load the required form into the memory, and then, use the Show method to display the form.

4.2.4. Design of the Login Module. In order to prevent illegal users from using the system, the system login module is provided in the server of the enterprise management system. The module is the portal for users to enter the system. Only through the login module can the user be authenticated and enter the main interface of the system. In order to verify whether the user name and password are correct, it is necessary to query the user information from the database according to the user name and password entered by the user. If the user information is correct, it will enter the main interface of the system; otherwise, there will be corresponding prompt information.

First, create a new form in the project, name it frm_xtdl, and set the StartUpPosition property to "2-screen center," the BorderStyle property to 0-None, and the Picture property to the specified picture. Second, add a PictureBox control on the form and set its AutoSize property to true, which is consistent with the size of the picture. Set the Picture property to the specified picture. Finally, add a ListView control and an ImageList control. These two controls are ActiveX controls, not Visual Basic standard controls, so you need to select the "Project"/"Parts" command and check the check box in the pop-up "Parts" Microsoft Windows Common Controls 6.0 (SP6) dialog box. Add the ListView control and ImageList control to the toolbox. Set the BorderStyle property of the ListView control to 0 -ccNone, and set the ListView control to have no border, and it can be embedded in the picture seamlessly, and at the same time, add pictures to the ImageList control. Through the above steps, the enterprise management system design is realized.

\section{System Test Results and Analysis}

5.1. Setting System Test Environment. In order to test the effectiveness of the enterprise management system based on the edge computing architecture, the system is tested and deployed in the WEB environment, and the system performance is tested through the enterprise's internal network and Internet client terminals. The system is a Windows 
Server 2018 server, configured as Intel Pentium $43.0 \mathrm{GHz}$ or higher dual CPU, $8 \mathrm{~GB}$ memory, SCSI dual hard disk mirroring, and SQL Server 2003. The configuration on the client is Intel Pentium 43.0 GHz or higher and WindowsXP/ Vista operating system. The test indicators are the proportion of system bugs, the system CPU occupancy rate, and the system response time. The method in $[4,5]$ and the proposed method are used to compare the system performance of the proposed method.

5.2. System Vulnerability Test and Analysis. Bug is in this system program development process; there are some hidden defects or problems that have not been found, that is, vulnerability. The higher the proportion of system bugs, the more system vulnerabilities. On the contrary, the lower the proportion of system bugs, the less the system vulnerabilities. In the system unit test phase, integration test phase, and acceptance test phase, the method in $[4,5]$ and the proposed method are used to test, respectively, and the number of system bugs of different methods is compared, as shown in Figure 4.

According to Figure 4, in the system unit test phase, integration test phase, and acceptance test phase, the system bugs of the method in [4] account for $32 \%, 28 \%$, and $21 \%$, respectively, and the system bugs of the method in [5] account for $39 \%, 33 \%$, and $42 \%$, respectively, while the system bugs of the proposed method account for $9 \%, 11 \%$, and $7 \%$, respectively. Therefore, the number of system bugs of the proposed method is relatively low, and the system vulnerabilities are less.

5.3. System Running Speed Analysis. CPU occupancy is the $\mathrm{CPU}$ resources occupied by running programs. The higher the CPU occupancy is, the slower the system runs. Conversely, the lower the CPU occupancy is, the faster the system runs. In order to verify the running speed of the enterprise management system based on the edge computing architecture, the number of clients is set to 400 , and the method in $[4,5]$ and the proposed methods are used to test, respectively. The CPU occupancy rate of different methods is compared, and the results are shown in Figure 5.

As can be seen from Figure 5, with the increase of the number of system clients, the CPU occupancy of the system with different methods increases. Among them, when the number of clients reaches 400 , the CPU occupancy rate of the method in [4] is $68 \%$, the CPU occupancy rate of the method in [5] is $43 \%$, and the CPU occupancy rate of the proposed method is only $21 \%$. Therefore, compared with the method in $[4,5]$, the CPU occupancy of the proposed method is lower and the running speed is faster.

5.4. System Response Time Analysis. On this basis, the response time of the enterprise management system based on the edge computing architecture is further verified. The method in $[4,5]$ and the proposed method are used to compare the system response time of different methods, as shown in Figure 6.

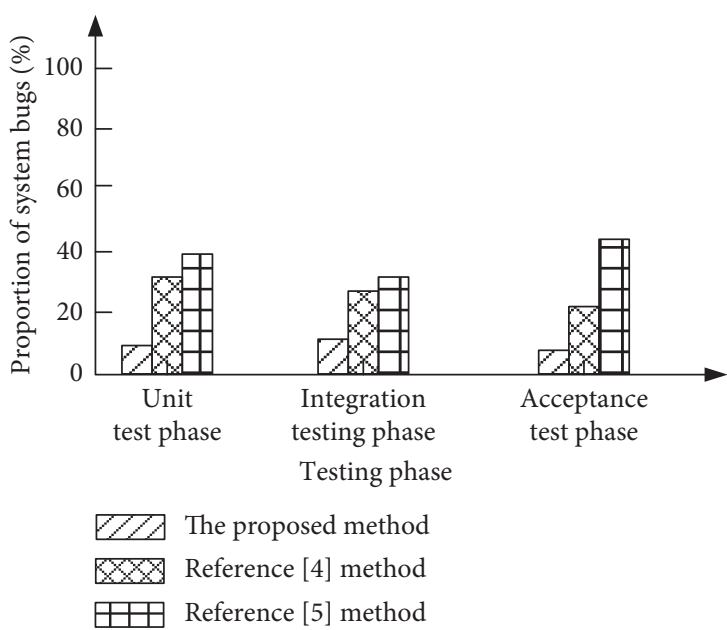

Figure 4: Comparison results of the number of system bugs by different methods.

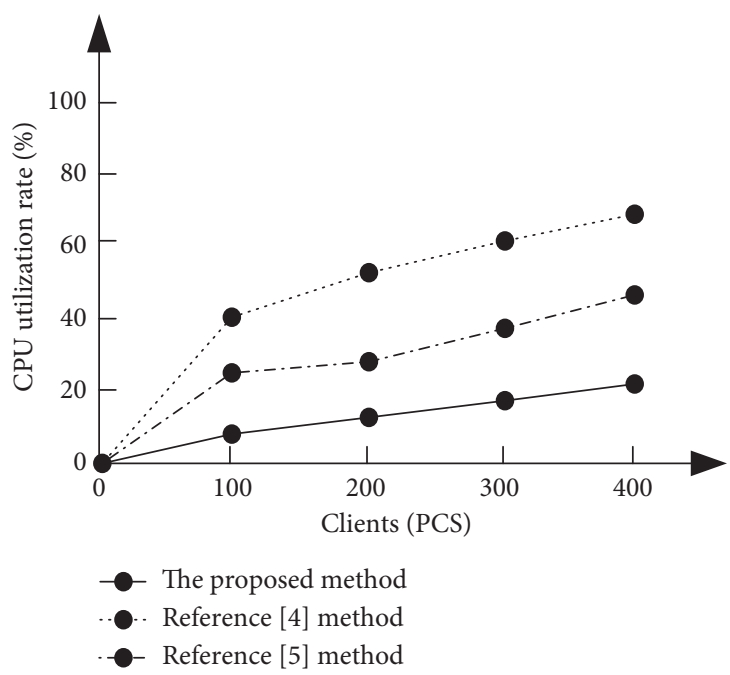

FIgure 5: Comparison results of system CPU usage of different methods.

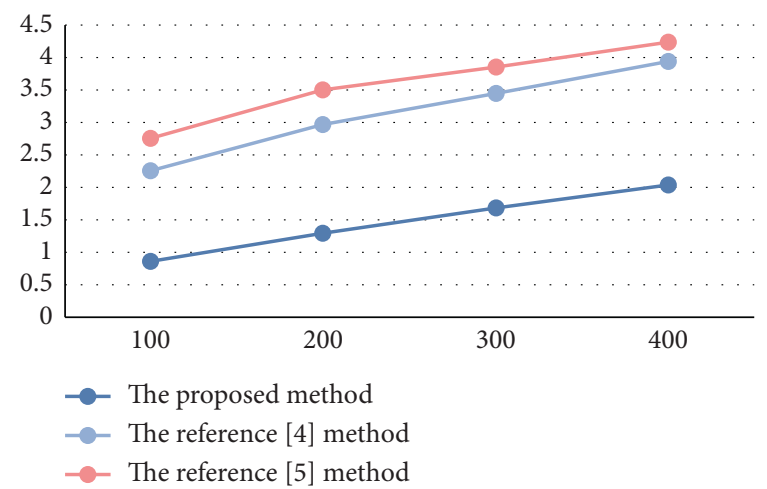

FIGURE 6: System response time comparison of different methods.

According to the data in Figure 6, with the increase of the number of clients, the system response time of different methods increases. When the number of clients is 400 , the system response time of the method in [4] is $3.95 \mathrm{~s}$, that of 
the method in [5] is $4.23 \mathrm{~s}$, and that of the proposed method is only $2.04 \mathrm{~s}$. Therefore, compared with the method in $[4,5]$, the system response time of the proposed method is shorter.

\section{Conclusion}

According to the characteristics of mobile edge computing, this paper designs an enterprise management system based on edge computing architecture, designs an edge computing platform architecture, integrates the MEC virtualization infrastructure layer, MEC platform layer, and MEC application layer, constructs an edge computing model, reflects the coupling relationship between enterprise management and operation and maintenance database, and realizes the parameter calculation of enterprise management and operation and maintenance system. Analyze the requirements of the enterprise management system, design the system function structure, database, and data table, and realize the design of the enterprise management system based on edge computing architecture. The system can effectively reduce the system vulnerabilities, speed up the system operation speed, and shorten the system response time. But in the enterprise management system, we should further improve the function of the system and verify the superiority of the system in practical application. Therefore, in the next step of research, the system is applied to specific engineering application projects to further verify the effectiveness of the enterprise management system.

\section{Data Availability}

The datasets used and/or analyzed during the current study are available from the corresponding author upon reasonable request.

\section{Conflicts of Interest}

The authors declare that there are no conflicts of interest.

\section{References}

[1] Y. Liu, F. R. Yu, X. Li, H. Ji, and V. C. M. Leung, "Decentralized resource allocation for video transcoding and delivery in blockchain-based system with mobile edge computing," IEEE Transactions on Vehicular Technology, vol. 68, no. 11, pp. 11169-11185, 2019.

[2] W. Li, Y. Wang, Q. Ren et al., "Design and implementation of digital control system based on PAC architecture for largecapacity pulse power supply," IEEE Transactions on Plasma Science, vol. 47, no. 12, pp. 5339-5344, 2019.

[3] C. Valor, C. Escudero, V. Labajo, and R. Cossent, "Effective design of domestic energy efficiency displays: a proposed architecture based on empirical evidence," Renewable and Sustainable Energy Reviews, vol. 114, no. OCT, pp. 109301.1-109301.11, 2019.

[4] M. S. Ravelomanantsoa, Y. Ducq, and B. Vallespir, "A state of the art and comparison of approaches for performance measurement systems definition and design," International Journal of Production Research, vol. 57, no. 15-16, pp. 50265046, 2019.
[5] T. Mäki and H. Kerosuo, "Design-related questions in the construction phase: the effect of using the Last Planner System in design management 1," Canadian Journal of Civil Engineering, vol. 47, pp. 290-382, 2020.

[6] A. J. Ferrer, J. M. Marquès, and J. Jorba, "Towards the decentralised cloud," ACM Computing Surveys, vol. 51, no. 6, pp. 1-36, 2019.

[7] M. Mehrabi, D. You, V. Latzko, H. Salah, M. Reisslein, and F. H. P. Fitzek, "Device-enhanced MEC: multi-access edge computing (MEC) aided by end device computation and caching: a survey," IEEE Access, vol. 7, pp. 166079-166108, 2019.

[8] M. Satyanarayanan, B. Paramvir, R. Caceres, and N. Davies, "The case for VM-based cloudlets in mobile computing," IEEE Pervas Computing, vol. 8, pp. 4-23, 2009.

[9] K. Bilal, O. Khalid, A. Erbad, and U. K. Samee, "Potentials, trends, and prospects in edge technologies: fog, cloudlet, mobile edge, and micro data centers," Computing Network, vol. 2017, 2017.

[10] M. Satyanarayanan, "The emergence of edge computing," Computer, vol. 50, pp. 1-30, 2017.

[11] M. Satyanarayanan, P. Simoens, Yu Xiao et al., "Edge analytics in the internet of things," IEEE Pervas Compututing, vol. 14, pp. 2-31, 2015.

[12] F. Bonomi, R. Milito, Z. Jiang, and S. Addepalli, "Fog computing and its role in the internet of things," in Proceedings of the 1st Edition of the MCC Workshop on Mobile Cloud Computing, Helsinki, Finland, August 2012.

[13] P. Garcia Lopez, A. Montresor, D. Epema et al., "Edge-centric computing," ACM SIGCOMM Computer Communication Review, vol. 45, pp. 5-42, 2015.

[14] Y. Ma, C. Lu, B. Sinopoli, and S. Zeng, "Exploring edge computing for multi-tier industrial control," IEEE Transactions on Computer-Aided Design of Integrated Circuits and Systems, vol. 39, no. 11, pp. 648-653, 2020.

[15] M. S. C. Inguva and J. B. Seventiline, "Implementation of FPGA design of FFT architecture based on CORDIC algorithm," International Journal of Electronics, vol. 18, no. 10, pp. 750-759, 2020.

[16] R. Zhang, J. Wu, R. Wang, R. Yan, Y. Zhu, and X. He, “A novel battery management system Architecture based on an isolated power/data multiplexing transmission bus," IEEE Transactions on Industrial Electronics, vol. 66, no. 8, pp. 5979-5991, 2019.

[17] C. Li, J. Tang, and Y. Luo, "Scalable replica selection based on node service capability for improving data access performance in edge computing environment," The Journal of Supercomputing, vol. 75, no. 11, pp. 7209-7243, 2019.

[18] D. Marmsoler and L. Eichhorn, "On the impact of architecture design decisions on the quality of blockchain-based applications," The Knowledge Engineering Review, vol. 35, pp. 193-199, 2020.

[19] B. Y. Kong, "Multi-touch detector architecture based on efficient buffering of intensities and labels," Electronics Letters, vol. 56, no. 1, pp. 884-892, 2020.

[20] P. Bellavista, D. Belli, S. Chessa, and L. Foschini, “A socialdriven edge computing architecture for mobile crowd sensing management," IEEE Communications Magazine, vol. 57, no. 4, pp. 68-73, 2019.

[21] L. Nie, "Visual design of marine architecture in coastal cities based on "BIM + VR"” Journal of Coastal Research, vol. 112, no. sp1, pp. 2112-2118, 2020. 
[22] F. Li, D. Li, and W. Li, "Research and design of load balancing algorithm for edge coverage network storage," Computer Simulation, vol. 37, no. 11, pp. 390-393, 2020.

[23] R. T. Tiburski, C. R. Moratelli, S. F. Johann et al., "Lightweight security architecture based on embedded virtualization and trust mechanisms for IoT edge devices," IEEE Communications Magazine, vol. 57, no. 2, pp. 67-73, 2019. 